\title{
Conservation of a glutamate residue in ATP-citrate lyase and succinyl-CoA synthetase
}

\author{
M.E. Fraser, J. Huang \\ Department of Biological Sciences, University of Calgary, 2500 University Dr. NW, Calgary, Alberta, T2N 1N4, Canada \\ frasm@ucalgary.ca
}

Succinyl-CoA synthetase (SCS), the enzyme that catalyzes the only substrate-level phosphorylation in the citrate cycle, is the prototype for a family of ADP- or GDP-forming acyl-CoA synthetases that includes ATP-citrate lyase (ACLY) [1]. These enzymes catalyze the formation of a thioester bond between an organic acid and CoA, using the energy of nucleotide triphosphate (NTP) and in the presence of magnesium ions. A histidine residue is transiently phosphorylated during catalysis [2], leading to the proposed catalytic mechanism:

$$
\begin{aligned}
\mathrm{E}+\mathrm{NTP} & \rightleftharpoons \mathrm{E}-\mathrm{PO}_{3}+\mathrm{NDP} \\
\mathrm{E}-\mathrm{PO}_{3}+\text { carboxylate } & \rightleftharpoons \mathrm{E} \cdot \text { carboxyl-phosphate } \\
\mathrm{E} \cdot \text { carboxyl-phosphate }+\mathrm{CoA} & \rightleftharpoons \mathrm{E}+\text { carboxyl-CoA }+\mathrm{P}_{\mathrm{i}}
\end{aligned}
$$

where E represents the enzyme; -, a covalent bond; and -, noncovalent interactions. For SCS, the carboxylate is succinate; for ACLY, it is citrate and there is fourth step in which citryl-CoA is cleaved to form acetyl-CoA and oxaloacetate.

A glutamate residue of ACLY, E599, was proposed to play a role in the cleavage of citryl-CoA [3]. This glutamate residue is conserved not only in ACLYs but also in SCSs (Fig. 1). The structures of SCSs and ACLYs found in the Protein Data Bank [4] are used to investigate the role of this conserved glutamate residue.

Human ACLY

Chlorobium limicola ACLY

Human GTPSCS $\alpha$-subunit

Escherichia coli $\alpha$-subunit

Thermus aquaticus $\alpha$-subunit

IRTIAIIAEGIPEALTRKLIKKA-DQKGVTI IGPATVGGIKPGCFKIGNTGGMLDNILASKLYR
IQLVSMITEGVPEKDAKRLKKLA-QKLGKMLNGPSS IGIMSAGECRLGVIGGEFKNLKLCNLYR
IPLVVCITEGIPQQDMVRVKHKLLRQEKTRLIGPNCPGVINPGECKIGIMPG-------HIHK
IKLIITITEGIPTLDMLTVKVKL-DEAGVRMIGPNCPGVITPGECKIGIQPG-------HIHK
IPLIVLITEGIPTLDMVRAVEEI-KALGSRLIGGNCPGI ISAEETKIGIMPG--------HVFK

Figure 1. Alignment of portions of the sequences of ACLYs and SCSs. The alignment shows conservation of a glutamate residue, E599 in human ACLY, E112 in the A-subunit of Chlorobium limicola ACLY, E105 $\alpha$ of human GTPSCS, E98 $\alpha$ of E. coli SCS, and E97 $\alpha$ of Thermus aquaticus GTPSCS.

[1] Sánchez, L. B., Galperin, M. Y. \& Müller, M. (2000). J. Biol. Chem. 275, 5794.

[2] Kreil, G. \& Boyer, P. D. (1964). Biochem. Biophys. Res. Commun. 16, 551.

[3] Wei, X., Schultz, K., Bazilevsky, G. A., Vogt, A. \& Marmorstein, R. (2020). Nat. Struct. Mol. Biol. $27,33$.

[4] Berman, H. M. et al. (2000). Nucleic Acids Res. 28, 235.

Keywords: ATP-grasp fold; phosphohistidine; magnesium ions; nucleotide 